\title{
Ganadería y unidades productivas mixtas en Buenos Aires colonial, 1726-1759
}

\author{
Mauro Pelozatto Reilly ${ }^{1}$ \\ Recibido: 15 de enero de 2016 - Aceptado: 15 de abril de 2016
}

\begin{abstract}
Resumen
Esta investigación se propone caracterizar las unidades productivas del Buenos Aires rural entre 1726-1759, desde la extinción del ganado vacuno cimarrón y las vaquerías, y la división de la campaña bonaerense oriental entre Buenos Aires y Montevideo, hasta la separación del espacio rural entre las jurisdicciones de los cabildos de Buenos Aires y Luján, y la consolidación de las distintas prácticas como recogidas de ganado y de la cría este tipo de hacienda en las estancias. A partir de estadísticas y descripciones sobre padrones rurales, sucesiones y testimonios del cabildo, se desarrollan y ponen en discusión temas como la evolución de la ganadería vacuna, la cría de otras especies, sus características y alternativas económicas, la relación con la agricultura y la existencia de distintos tipos de unidades productivas. Se sostiene la idea de que existieron múltiples posibilidades para la ganadería diversificada y las explotaciones mixtas en los mercados coloniales.
\end{abstract}

Palabras clave: Ganadería, unidades productivas, producción agrícola, mercados coloniales.

\section{Livestock and mixed production units in colonial Buenos Aires, 1726-1759}

\begin{abstract}
This research aims to characterize the production units at rural Buenos Aires between 1726 and 1759, from extinction of bighorn cattle and dairy farms and the division of the eastern Buenos Aires province between Buenos Aires and Montevideo, to the separation of rural space between jurisdictions of the town halls of Buenos Aires and Lujan and the consolidation of the different practices as collecting and breeding livestock in such farms. From statistics and descriptions of rural records, inheritances and testimonies from the town halls, the study develops and discusses issues such as the development of
\end{abstract}

Argentino. Profesor en Historia, Universidad de Morón, Argentina. Especialista en Ciencias Sociales con mención en Historia Social por la Universidad Nacional de Luján. Actualmente se encuentra realizando la tesis de Maestría en Ciencias Sociales, mención en Historia Social, en dicha institución. Docente en la Universidad de Morón y la Universidad Nacional de La Matanza. E-mail: maurolpr@hotmail.com.ar 
beef cattle, breeding of other species, their characteristics and economic alternatives, the relationship with agriculture and the existence of different types of production units. The idea is that there were many opportunities for diversified livestock and mixed farms in colonial markets.

Keywords: Livestock, production units, agricultural production, colonial markets.

\title{
Pecuária e unidades de produção mistas em Buenos Aires colonial, 1726-1759
}

\begin{abstract}
Resumo
Esta pesquisa tem como objetivo caracterizar as unidades de produção de Buenos Aires rural entre os anos 1726-1759 rural, desde a extinção do gado de carne de vaca selvagem e as leiterias, e a divisão da campanha bonaerense oriental entre Buenos Aires e Montevidéu, até a separação do espaço rural entre as jurisdições dos cabildos de Buenos Aires e Luján e a consolidação das diferentes práticas como a coleta de gado e da cria, neste tipo de fazenda nas estâncias. A partir de estatísticas e descrições de padrões rurais, sucessões e testemunhas dos cabildos, se desenvolvem e colocam em discussão temas como a evolução do gado, da cria de outras espécies, suas características e alternativas econômicas, a relação com a agricultura e a existência de diferentes tipos de unidades de produção. Se mantem a ideia de que havia muitas possibilidades para a pecuária diversificada e as explorações mistas nos mercados coloniais.
\end{abstract}

Palavras-chave: unidades de produção pecuária, produção agrícola, mercados coloniais.

\section{La ganadería durante el siglo XVIII: un repaso desde el análisis de las unidades productivas}

La ganadería, y sobre todo la practicada sobre el ganado vacuno, fue sufriendo importantes transformaciones durante la primera mitad del siglo XVIII. A partir de la extinción del ganado cimarrón y con este de las vaquerías tradicionales ${ }^{2}$, los vecinos criadores tuvieron que aumentar la cría de ganado en sus tierras, necesariamente, para poder disponer de este recurso. Además, se fueron consolidando otras formas de explotación como las recogidas de alzados para repoblar estancias y las faenas sobre los abundantes planteles 
de vacunos salvajes que todavía quedaban en la Banda Oriental del Uruguay ${ }^{3}$ (Pelozatto Reilly, 2015a; 2015b), las cuales es preciso indagar y desarrollar por separado. El objetivo de este artículo se centra en analizar las unidades productivas (UP) dedicadas a la cría de ganados, con el fin de apreciar, entre otras cosas, la importancia del vacuno dentro de las mismas en este período de cambios para la actividad pecuaria. Asimismo, se parte de la base de que las vacas y toros no fueron las únicas alternativas mercantiles, para las prácticas ganaderas en Buenos Aires colonial. Además de tener en cuenta, como lo han demostrado algunas investigaciones, que la estancia nació en esta región vinculada directamente a la producción de mulas para abastecer al Alto Perú y la industria argentífera (Azcuy Ameghino, 1995; Garavaglia, 1999), estaba la cría de otras especies que pudieron haber respondido a otras necesidades mercantiles y de consumo, como los mulares, equinos, bueyes y ovinos. A su vez, hablando del vacuno, se plantea la idea de que la cría en las estancias y demás UP no vino a suplantar a las extintas vaquerías, sino que ya estaba presente desde antes (González Lebrero, 1993), pero a partir de dicho proceso aumentó su número y concentración en las extensiones de tierras rurales.

Antes de intentar describir a las unidades productivas es preciso definir qué se entienden aquí como tales. En este punto, se toma la definición utilizada por Juan Carlos Garavaglia, para quien estas eran "los establecimientos productivos -que manteniendo un grado de autonomía relativa entre sí- engloban en su seno tanto a grupos domésticos como a trabajadores dependientes si los hubiese" (Garavaglia, 1999). Asimismo, también se seguirá la postura de que en la campaña bonaerense existieron diferentes clasificaciones para éstas, tales como las de estancias y chacras, por ejemplo, las cuales supieron tener ciertas características que las diferenciaban unas de las otras así como también a quienes las ocupaban. Es acertado tomar ciertas precauciones a la hora de hacer estadísticas y descripciones a partir de estas clasificaciones ya que, como se detallará más adelante, las fuentes disponibles pueden traer consigo ciertas debilidades que pueden convertirse en las del historiador y, además, los testimonios de la época ponen de manifiesto una zona rural en la cual no todas las chacras ni las estancias eran iguales entre sí, ni mucho menos.

Tanto la evolución de la ganadería como la organización interna de las unidades de producción y trabajo rurales son temas que han sido tratados, discutidos y problematizados de diferentes maneras, perspectivas y a partir del estudio de diversas fuentes históricas. Si bien no se hallan trabajos co-

Territorios que pertenecían a Buenos Aires hasta la fundación del cabildo y ciudad de Montevideo (1726), que vino a dividir las campañas orientales en dos jurisdicciones. 
rrespondientes a la primera mitad del siglo XVIII exclusivamente, ni tampoco para el recorte cronológico que se toma en esta investigación, es conveniente hacer un breve repaso historiográfico sobre el tema de las UP y cómo estas han sido abordadas, para a partir de allí poder extraer ciertas categorías, conceptos y metodologías que puedan servir para desarrollar lo que corresponde a este período y poner en discusión con la bibliografía y las fuentes algunos puntos importantes.

Desde hace algunos años a esta parte, se han realizado importantes aportes en cuanto a la estancia y demás UP en Buenos Aires colonial. Tomando la primera mitad del siglo XVII, Rodolfo González Lebrero se ocupó de caracterizar las chacras y estancias en base a los datos ofrecidos por 20 testamentos y 9 inventarios para el período 1602-1640. Haciendo un análisis cuantitativo y cualitativo, el autor pudo reconstruir las características de los medios de producción y la fuerza de trabajo utilizados en los establecimientos, la distribución de los distintos tipo de unidades por pagos ${ }^{4}$ y por regiones, la presencia de prácticas agrícolas en establecimientos más orientados a la cría (usando como indicadores el hallazgo de herramientas representativas de dicha producción y molinos, por ejemplo), cuantificó la importancia de las distintas especies de ganado (vacas, ovejas, cerdos, etc.), describió las orientaciones mercantiles de la actividad pecuaria (cueros, grasa, sebo, etc.), y encontró otras actividades como los textiles y la elaboración de vino. Otros puntos destacables son haber calculado el número de esclavos y la distribución general del valor de los bienes que se encontraron dentro de los establecimientos (González Lebrero, 1993). Entre tantas virtudes, creo que este estudio tiene dos puntos débiles, como el recorte temporal que no fue justificado debidamente, y un corpus documental bastante acotado para el período que se buscó abarcar.

Carlos Mayo y Ángela Fernández realizaron una "anatomía" de la estancia (1750-1810), concentrándose en el análisis de inventarios y tasaciones de bienes de 66 establecimientos. En base a estos datos, desarrollaron una investigación fundamentalmente integrada por estadísticas y porcentajes sobre los tipos de ganado, qué difundida estaba cada especie, cuáles eran los posibles mercados para sus productos, qué valor representaban la tierra y los esclavos, en qué tipo de construcciones edilicias e instalaciones invertían los estancieros, qué tecnología utilizaban para las prácticas agropecuarias, etc. Lo que más se destaca de artículos como este es la estructura, que es de utilidad para analizar elementos similares en otros períodos como el del correspondiente artículo (Mayo y Fernández, 1993). Tomando una cronología (1750-1850) y estructura de exposición similares, Garavaglia

Jurisdicciones rurales que formaban parte de la del cabildo de Buenos Aires. 
hizo un trabajo sobre las estancias de la misma jurisdicción y sus medios de producción, destacándose por trabajar el mismo tipo de fuentes de diferente manera: en primer lugar, el autor realizó una descripción de la documentación (inventarios) mucho más sólida, así como también de la metodología a implementar; en segundo término, supo elaborar-sacando promedios con los registros consultados- un "establecimiento típico" (modelo de análisis), con determinada extensión territorial (2.500 hectáreas) y composición pecuaria (790 vacunos, 490 ovinos, 300 equinos, 40 mulares y 12 bueyes). También realizó un detallado análisis de la evolución del stock ganadero del período por regiones, además de identificar y caracterizar desde descripciones y estadísticas a los pequeños, medianos y grandes propietarios, pudiendo destacar la importancia numérica de los dos primeros grupos por sobre los terratenientes hacendados (Garavaglia, 1993). En un trabajo más reciente, Carlos Mayo, partiendo desde la caracterización de las estancias, desarrolló capítulos dedicados al ganado pero también a los distintos grupos sociales (estancieros, agregados, peones, esclavos, campesinos libres, mujeres y administradores de estancias), sus hábitos, los conflictos sociales, sus condiciones de trabajo y de vida, la relación con los establecimientos, etc. Resulta interesante cómo se pueden desarrollar diferentes temas partiendo de las UP y triangulando distintos tipos de fuentes (Mayo, 2004).

Por otro lado, están los conocidos como estudios de caso, que bien sirven para ilustrar algunas cuestiones y ponerlas en debate con las fuentes tomadas para este escrito. Tulio Halperín Donghi sintetizó de manera excelente el funcionamiento y la organización interna de la estancia colonial desde la administración betlemita en Fontezuela (1753-1809), revisando los libros de cuentas y transacciones comerciales desde y hacia dicha UP. Pudo apreciar claramente la importancia de la mano de obra esclava, su relación de complementariedad con los asalariados, los productos adquiridos por la estancia y la distribución de los mismos, y la producción que allí se daba para salir hacia los distintos mercados, definiendo que una unidad que criaba diferentes especies animales y producía efectos varios se relacionaba con más de un mercado (Halperín Donghi, 1993). Existen otros artículos y capítulos dedicados a otro tipo de UP, las orientadas más hacia la agricultura del cereal. Jorge Gelman realizó un análisis regional de los establecimientos de la Banda Oriental hacia fines de la colonia, caracterizando las condiciones de producción en el caso de una chacra ubicada dentro de una estancia, los costos representados por la empresa agrícola y los tipos de mano de obra utilizados para luego hacer una cuantificación de la producción e ingresos registrados (Gelman, 1993a). Por su parte, Raúl Fradkin estudió la hacienda de la Chacarita (1779-1784), desde los inventarios y tasaciones a los cuales explotó muy bien para poder alcanzar consideraciones importantes sobre los esclavos y su valor, los distintos tipos de edificios, las actividades productivas 
diversificadas, las situaciones de arrendamiento registradas ${ }^{5}$, los gastos e ingresos de la hacienda, rasgos de la actividad triguera y su relación con el arriendo, y comparaciones en número y producción entre esclavos, peones y arrendatarios, entre otras cosas (Fradkin, 1993b).

Siguiendo con el repaso de los estudios en escalas reducidas, hay que señalar que otros se dedicaron a problemáticas más específicas en torno a las UP. Citando un ejemplo, Carlos Birocco se centró en un establecimiento (el de don Miguel de Riblos y sus sucesores en Areco) para tratar otras temáticas como la importancia del latifundio en Buenos Aires colonial, la ganadería diversificada y sus alternativas comerciales, la mano de obra y las formas de pago, las estrategias empleadas por los distintos actores para el ascenso socioeconómico, entre otras, poniendo en juego fuentes como sucesiones, testamentos, protocolos, libros de cuentas y cartas de administradores de estancias (Birocco, 1996).

Es necesario extraer problemas y elementos de todos estos trabajos señalados para intentar desarrollarlos en el período 1726-1759: en primer lugar, el uso de las fuentes como sucesiones (inventarios, testamentos, tasaciones de bienes) que serán complementadas con padrones rurales y otros testimonios; a su vez, se pondrán a discutir con las fuentes las características internas de las UP, su organización interna, la composición de las haciendas ${ }^{6}$, las orientaciones mercantiles, y la relación entre las diversas prácticas pecuarias y agrarias; también es de utilidad rescatar las estructuras de algunas investigaciones que se hicieron para la región seleccionada, como las de Carlos Mayo y Juan Carlos Garavaglia anteriormente citadas, así como también es preciso no descuidar las particularidades locales y de casos puntuales que puedan aparecer. Creo que no es acertado desarrollar tantas problemáticas en un artículo y en un período de varias décadas, como lo hicieron los autores mencionados, y es por eso que se ha decidido dejar afuera de este análisis a cuestiones como las distintas fuerzas de trabajo, las relaciones sociales, las condiciones de vida de los ocupantes y propietarios, los vínculos personales y grupales en torno a la tierra y el ganado, entre otras que es conveniente estudiar más intensamente por separado. Por eso, esta investigación se centra en las características de las UP en cuanto a ganado se refiere, y las distintas prácticas pecuarias en relación a las diversas demandas para sus productos.

Se entienden como relaciones consuetudinarias en las cuales el propietario de la tierra ofrecía una porción o parcela a un pastor o labrador a cambio del pago de un tributo que podía darse de diferentes formas.

6 Se emplea el término "hacienda" entendida como ganado y la posesión del mismo por parte de las UP. 


\section{El ganado}

El ganado representaba el componente más importante para las UP, tanto por su cantidad como por su difusión y su valor monetario dentro de las mismas, al igual que lo sería en el período posterior a este (Mayo, 2004: 40). Tomando como referencia los censos ${ }^{7}$ rurales del período, únicamente los de 1738 y 1744 permiten considerar las cantidades de ganado en las UP y el número de éstas que tenían haciendas. En el primer caso, se registraron 1.023 unidades diferenciadas, de las cuales 416 poseían animales (40,66\%) (ANH, Padrón de 1738: 288-327), mientras que en el segundo caso, sobre 891 explotaciones, solo 103 aparecen en este rubro (11,56\%) (ANH, Padrón de 1744: 509-709). Empero, los padrones no resultan una fuente contundente para demostrar lo que se busca, debido a que la misma puede estar sujeta a puntos débiles como, por ejemplo, que en aquel momento no haya sido del interés del cabildo (mandaba a levantar los padrones) o de los empadronadores (generalmente alcaldes de la hermandad) registrar los ganados con especificidad, sino que tenían otros objetivos para la realización del padrón como contar los hombres disponibles para las levas de las milicias de fronteras o para reclutar gente y formar nuevas poblaciones, como fue el caso del correspondiente al año 1726, el cual no fue siquiera citado en este trabajo porque no aparecen anotados los animales, ya que el objetivo era reclutar y mandar familias a la nueva ciudad de Montevideo.

Por eso es que resulta necesario complementar esta fuente secundaria con una primera como las sucesiones (inventarios de chacras y estancias, tasaciones de bienes, testamentos, etc.): tomando una muestra significativa de 81 casos encontrados para el período 1726-1759, 58 tenían ganado $(71,6 \%)$, tomando al mismo como indicador directo y sin considerar las UP que bien podrían ser de cría por su extensión territorial y otros indicios como la posesión de piezas de cueros, sebo, grasa y corrales (AGN, Tribunales, Sucesiones ${ }^{8}$ ). Esto nos da como claro panorama una importante divulgación de la cría de ganados sobre la muestra de estos registros un tanto más confiables 9 . Ahora es necesario especificar más sobre la ganadería a partir de estos datos.

Se utiliza como sinónimo de padrón.

8 Las estadísticas señaladas en este estudio han sido elaboradas a partir de datos extraídos de los legajos $n^{0} 3859,5335,5336,5337,5338,5671,6253,6254,6370,6722,6723,6725,8122,8130$ y 8417.

9 En este punto es importante hacer una precisión. Tanto en las unidades registradas por los padrones como en las sucesiones, cuando en los casos aparece mencionada la presencia de algún tipo de ganado, pero no se especifica su cantidad exacta, se consideró como si se tratara de una cabeza, para no incluir cifras ficticias. Por lo tanto, cada valor representa un mínimo del total real que no pudo ser cuantificado. 


\section{La ganadería vacuna y sus variantes}

Respecto al ganado vacuno, que es lo que nos interesa seguir en esta investigación ${ }^{10}$, el mismo era muy importante para los establecimientos de cría. Esto puede verse tanto en los padrones rurales como en las sucesiones. Según los primeros, en 1738 las UP con vacas y relacionados totalizaron 244 , sobre un total de 416 dedicados a la cría $(58,65 \%)$, y en 1744 , de 104 , unas 86 contaban con este tipo de producción (82,69\%) (ANH, Padrones de 1738 y 1744: 288-709). En definitiva, la mayoría de las tierras dedicadas a la ganadería contaban con vacunos, lo cual puede considerarse también luego de ver los inventarios, testamentos y tasaciones: sobre el total (81), 58 estaba vinculados a las prácticas pecuarias, entre las cuales 42 tenían vacunos (72,41\%) (ANH, Padrones de 1738 y 1744: 288-709), dando una tendencia similar que si tomamos el mismo indicador con distintas fuentes.

A este tipo de cría se le presentaban diferentes posibilidades en los mercados coloniales, como lo eran el abasto de carne para la población local, la producción de cueros para exportar por el Atlántico, la recolección de animales para ser enviados en pie hacia otras regiones como Chile y el Alto Perú, y las faenas para elaborar sebo y grasa (Garavaglia, 1999: 216-217; Mayo, 2004: 41). Esto puede apreciarse gracias a algunos datos brindados por las sucesiones y los testimonios extraídos del cabildo porteño.

En las sucesiones pueden hallarse algunos elementos como, por ejemplo, los cueros que tenía almacenado don Miguel de Arismendi, además de varias piezas de sebo y grasa (AGN, Tribunales, Sucesiones, 3859), o los 21 cueros que se le contaron a Joseph Carrizo, valuados a 4 reales cada uno (AGN, Sucesiones, 5337). Por otra parte, el cabildo le dio mucha importancia a la producción y comercialización de cueros durante el período, y por eso gracias a algunos de sus testimonios podemos aproximarnos a las cantidades que solían manejarse por aquellos tiempos: por ejemplo, en 1739, se trató sobre los 32.000 que pidió Francisco de Alzaybar para cargar en sus navíos ese año (AGN, AECBA, Serie II, Tomo VIII: 46); ese mismo año, reconocieron obediencia a la Real Cédula que le permitía a Alzaybar hasta 15.000 cueros, mientras que se contabilizaron, por otra parte, los embarcados en los navíos del Real Asiento de Gran Bretaña, dando un total de 71.388 cueros (AGN, AECBA, Serie II, Tomo VIII: 101-102); más adelante, en 1750, unos comerciantes exigieron con el apoyo de una carta del director de la Compañía de Sevilla permiso para que se mandara a hacer corambre desde el ayuntamiento

10 Esta investigación forma parte de una más extensa para presentar como tesis para la obtención de Maestría en Ciencias Sociales con mención en Historia Social (UNLu), cuyo eje se centra en el cabildo de Buenos Aires, su intervención sobre la ganadería vacuna, y cuáles fueron las formas que esta última fue adoptando a lo largo del mismo período (1726-1759). 
entre los vecinos de la ciudad, y poder cargar entre 14 y 16.000 unidades en sus navíos (AGN, AECBA, Serie II, Tomo IX: 618-620); al año siguiente, se mencionó que don Pedro de Lea y su compañía habían negociado en Colonia por 31.032 cueros, que se sumaron a los 40.000 que totalizaban los que ya tenían cargados en sus barcos (AGN, AECBA, Serie III, Tomo I: 46-47).

A lo largo del período analizado, la ganadería vacuna fue atravesando importantes cambios. Hasta comienzos de la centuria, las vaquerías tradicionales seguían siendo la forma de explotación por excelencia del vacuno, con lo cual no se busca sostener que no existiera la cría en las UP (Fradkin, 2000; Harari, 2003; Pelozatto Reilly, 2015a). Las características de aquellas prácticas, que imponían la caza del ganado cimarrón ${ }^{11}$ por sobre la cría condujeron inevitablemente a su extinción (Halperín Donghi, 2010: 41), pese a los intentos del ayuntamiento por regular la situación con el nombramiento de vecinos "accioneros" y la concesión -o negación, según el caso- para hacer corambre o faenas de sebo y grasa (Birocco, 2003; 2015).

A partir de la desaparición de esas expediciones de caza, la ganadería vacuna fue adoptando nuevas formas como las recogidas de ganado alzado en los campos bonaerenses, las vaquerías sobre los abundantes planteles disponibles en la Banda Oriental, tanto para hacer faenas como para conseguir animales destinados al abasto y repoblar las estancias de este lado (Garavaglia, 1999: 216; Pelozatto Reilly, 2015b). Asimismo, durante el período que se está desarrollando, tuvieron lugar acontecimientos y procesos que contribuyeron a volcar la producción de los mercados regionales hacia Buenos Aires y su puerto con salida al Atlántico. Me refiero a lo que Vilma Milletich define como "atlantización" de la economía colonial, como consecuencia de la imposición de las potencias ultramarinas como Holanda, Francia y Gran Bretaña por sobre España y Portugal, ya desde mediados del siglo XVII, lo cual llevó a que las regiones tuvieran que producir en función de las nuevas demandas y se mantuvieran vinculadas directa o indirectamente con Europa (Milletich, 2000: 217-218). En el caso de Buenos Aires, esto se tradujo en un crecimiento del mercado de cueros y del comercio atlántico, lo cual ya fue demostrado por especialistas como Fernando Jumar, a partir no solamente de las cantidades de cueros exportados, sino también con el aumento de las entradas y salidas de navíos comerciales (Jumar, 2008).

Podría hacerse un paralelo entre la evolución del stock ganadero según los padrones y de la producción-exportación de cueros en este período de supuesta "atlantización" de la economía agropecuaria. Tomando la información de las UP de 1738 y 1744, en este último se ve un crecimiento en

11 Se está hablando del ganado vacuno salvaje que transitaba libremente por la campaña. 
el porcentaje de establecimientos con ganado vacuno en relación al total de unidades de cría (pasó del 40,66\% al 82,69\% en recorrido de pocos años). Además, si bien el número de explotaciones registradas es más bajo, la concentración de animales de este tipo crece. Por otra parte, observando las cantidades de vacunos registradas, puede sostenerse tal crecimiento de la producción de efectos pecuarios: en 1738 nos encontramos con 30.077 cabezas aproximadamente, promediando 123,26 cabezas por unidad sobre el total de UP que contaban con este tipo de actividades; en el padrón siguiente se han podido considerar 14.886 cabezas, dando un promedio de 173 por UP. Por lo tanto, podría deducirse que durante el período fue creciendo la concentración de ganado vacuno en las UP (ANH, Padrones de 1738 y 1744 : 288-327; 509-709) que se dedicaban a la cría del mismo, tendencia que quizás empezó desde antes en el período pero que los datos del 1726 no pueden demostrar del todo porque no registraron las haciendas. Otro indicador es el aumento de la exportación de cueros, el cual fue demostrado por Jumar para sostener el primer boom en la exportación de dicho producto: entre 1724 y 1758 los cueros exportados ${ }^{12}$ desde el complejo portuario rioplatense (considerando los circuitos de comercio español, portugués e inglés) representaron 2.897.838,887 pesos de a 8 reales (Jumar, 2008: 32); en unidades exportadas, la cantidad fue de 1.792.977 cueros exportados (Jumar, 2008: 34), niveles muy superiores a los anteriores a este período (Jumar, 2008: 31-34).

A su vez, otro elemento que puede servir para explicar el crecimiento de la concentración de vacunos por UP en este período puede ser el crecimiento demográfico, tal vez como consecuencia de la mayor importancia que fue adquiriendo a nivel comercial Buenos Aires y su puerto. Jumar nos habla de un crecimiento notable de la exportación de cueros desde la década 1740, momento desde el cual también comenzó a crecer considerablemente la población bonaerense: según el padrón de 1744, la zona Buenos Aires contaba con 6.035 habitantes, mientras que en 1778 había ya 17.000 almas aproximadamente (Mayo, 2004: 31). Ante mayor población, es lógico pensar en la necesidad de más novillos y vacas para el abasto de carne local, la cual fue otra de las principales preocupaciones del cabildo. Entre otras cosas, se encargaba de sacar a remate el derecho de abasto anual entre los vecinos criadores, de designar encargado cuando no se encontraban postores por causas como la escasez de ganado, de regular las matanzas y faenas con el fin de garantizar el alimento para la gente de su jurisdicción, controlar el ganado de las estancias de sus territorios y lo que se debía hacer con él, así como también de especificar qué hacer con las cabezas recogidas y vaquea-

12 Los recortes cronológicos realizados por el autor no coinciden exactamente con el realizado para esta investigación. Es por eso que se tomó un período algunos años más extendido (1723-1759), para analizar las tendencias respecto a las exportaciones de cueros desde el Río de la Plata. 
das, según el caso (Garavaglia, 1994; Dupuy, 2010; Pelozatto Reilly, 2015a). Además, era el principal organismo político local a la hora de controlar los corrales y el matadero, poner límites a los abastecedores y corraleros, fijar la frecuencia de las matanzas y hasta de acordar los precios por cuarto de res (AGN, AECBA, Serie II, Tomos VIII y IX; Serie III, Tomo I).

Por lo general, se prefería novillos y terneros para el consumo de carne, los cuales se ven como muy difundidos en los establecimientos ganaderos junto con las vacas, los que no eran descartadas como segunda instancia para el consumo (eran más importantes para la extracción de cueros). En las tierras que habían sido de don Miguel de Riblos, se encontraron, dentro de un stock ganadero enorme y diversificado, 410 terneros acompañando a las varias centenas de vacas (AGN, Sucesiones, 8122); Joseph de Esquivel tenía diferenciadas a sus vacas de novillos y terneras (AGN, Sucesiones, 5671); don Lorenzo Rodríguez contaba con 50 vacas y 50 terneras por lado, más aproximadamente 1.000 cabezas entre vacunos grandes y chicos (AGN, Sucesiones, 8130); doña María Ayala, entre otras haciendas, tenía 361 vacunos y 166 terneras (AGN, Sucesiones, 3859), entre otros casos. En cifras, la tendencia es más que clara: de las 42 unidades con ganado vacuno tomadas de las sucesiones, 25 (59,52\%) lo tenían claramente separado en vacas por un lado y terneras, novillos y menores de un año por el otro, ante lo que podría pensarse que los mantenían para distintos fines comerciales. Hay casos destacados tanto por la clasificación de los animales como por las cantidades: el capitán Ramón López, del pago de La Matanza, contaba entre sus vacunos con 2.500 vacas de cría, 100 lecheras mansas y 1.075 terneras (AGN, Sucesiones, 6722). De esta forma, vemos como dentro de este tipo de hacienda se presentaban varias alternativas en los mercados coloniales locales (carne), regionales (reses) y atlánticos (cueros).

\section{La cría de mulas y los mercados del Norte minero}

Otra especie de fundamental importancia era el ganado equino (caballos pero sobre todo las yeguas de cría), por ser piezas centrales en la fabricación de mulas. La obtención de dicho híbrido implicaba un proceso costoso y arriesgado que suponía el cruce entre las yeguas madrinas y los burros "hechores"13, lo cual se conseguía gracias a los caballos "retarjados", aquellos que habían sido operados para entrar en celo con las yeguas, pero no para poder preñarlas. A su vez, éstas eran acostumbradas a convivir con el burro desde que este era pequeño (se le colocaba el cuero de un potrillo muerto para que las hembras lo reconocieran con ese olor). En definitiva, una tarea

13 Burros dedicados a reproducirse con las yeguas madrinas para dar como resultado las crías de mulares. 
poco agradable y muy riesgosa, sobre todo una vez nacidas las crías, muy sensibles a los bruscos cambios de temperatura que no eran nada extraños en la campaña (Garavaglia, 1999: 210-211).

Estos animales eran vitales para la producción y el transporte de la plata que se concentraba en el Potosí y el Alto Perú, por lo que era vital el mantenimiento de una ruta entre aquella región y la ciudad-puerto de Buenos Aires con su campaña (Moutoukias, 1988: 21), sobre todo teniendo en cuenta que más allá de que esta jurisdicción formara parte del "espacio peruano", caracterizado por la especialización regional en función de las demandas de consumo efectuadas por el eje Lima-Potosí (Assadourian, 1983: 270-273; Fradkin y Garavaglia, 2009: 65), sino porque además hasta finales del siglo XVIII -por lo menos-, la plata era el principal producto de exportación tomando las salidas del puerto de Buenos Aires, rozando el $80 \%$ del total de las mismas (Halperín Donghi, 2010: 40).

De esta manera, podríamos sostener que el comercio de mulas conectaba regiones, desde Buenos Aires hasta el Norte minero y la capital del Virreinato, pasando por los distintos centros de producción y abastecimiento de mulas del interior del espacio (Paz, 1999: 45). Esta importancia se traducía hacia el interior de las UP rurales, donde se destacaban las importantes manadas de yeguas y el uso de caballos y burros hechores. Según los padrones de 1738, 121 UP tenían yeguas de cría (representando el $29 \%$ en relación al total de los establecimientos de cría) (ANH, Padrón de 1738: 288-327), mientras que en el registro siguiente vemos que solo 26 contaban con este tipo de reproductoras (ANH, Padrón de 1744: 509-709). En cuanto a la concentración, la misma es de 73,88 cabezas por UP en el último caso, mientras que en el primero (el más confiable por la cantidad de explotaciones contadas), es de 140 por sitio (ANH, Padrón de 1738: 288-327).

Tomando los inventarios, testamentos y tasaciones, uno se halla frente a un panorama similar: sobre 36 UP con animales vinculados a la cría de mulas $^{14}$, el promedio de animales es de 361 por cada una (13.000 animales registrados en total) (AGN, Sucesiones). Sin dudas, se trata de una cifra cercana a lo extraordinario, más teniendo en cuenta que los valores que se obtienen de fuentes como estas siempre son estimaciones menores al total real por varias cuestiones (como por ejemplo, la falta de datos por parte de los padrones rurales, o la falta de especificidad en el conjunto de las sucesiones, más otros detalles a tener en cuenta, como que no todos los documentos fueron realizados en la misma época del año y que, en el caso de las mulas, éstas solían partir hacia los mercados del Norte en el verano).

14 La fuente en este caso permite considerar yeguas, burros y mulas en los establecimientos, a diferencia de los padrones, donde solo se encontraron yeguas. 
Resultan llamativos también algunos ejemplos de grandes productores de mulas, que llegaban a constituir considerables haciendas de este tipo. Por ejemplo, Julio Gutiérrez de Paz tenía en sus estancias de Arrecifes, hacia 1738, 1.000 yeguas de cría junto con la misma cantidad de vacas, al igual que don Diego de Santa Ana y don Bernardo de Quintana, mientras que don Fernando de Quintana acumulaba 2.000 cabezas en sus tierras del mismo pago (ANH, Padrón de 1738: 316), siendo ésta la cantidad máxima registrada ese año. En los datos de 1744, se destacan los casos de Tomás de Arroyo, quien poseía 1.500 yeguas más 3.000 ovejas entre su estancia y su chacra del pago de Magdalena (ANH, Padrón de 1744: 701), y doña Martina de Luola, vecina del mismo pago que contaba con 1.000 animales de esta clase (ANH, Padrón de 1744: 709). Aquí sale a la luz otro rasgo a tener en cuenta, que es la posesión de importantes cantidades de distintas especies en el mismo establecimiento.

En los testamentos e inventarios, las cifras son todavía más abultadas, pese a que en la mayor parte de los casos, los productores de mulas también aparecen en pequeñas cantidades. Es valioso apreciar casos como el de don Miguel de Riblos, quien tenía entre sus tierras de Areco, además de ganado vacuno en grandes planteles y demás especies, 4.955 yeguas, 66 burros hechores (más otros que se mencionan pero que no fueron contados con exactitud), y 1.060 mulas (AGN, Sucesiones, 8122). Don Felipe Castro declaró 825 yeguas, 11 hechores y 97 mulas (AGN, Sucesiones, 5335). La viuda de López Osorio (1742) contaba con 907 yeguas y 25 mulas (AGN, Sucesiones, 5336). Don Tiburcio Casco poseía entre sus haciendas 502 yeguas, 10 burros reproductores y 129 mulas (AGN, Sucesiones, 5338). Don Bernardino de Ávalos tenía 610 yeguas de cría, 6 burros y 3 mulas (AGN, Sucesiones, 6722). Casos como estos ayudan a ver otros aspectos de este tipo de prácticas: la gran cantidad de yeguas en relación a los burros hechores (solo se necesitaban unos pocos para preñar a manadas enteras) y las mulas (las cantidades de crías obtenidas no solían ser muchas, y la mayoría marchaba pronto de las estancias hacia las ferias e invernaderos de mulares importantes como Córdoba y Salta).

A nivel regional también tuvieron peso, ya que las mulas que partían desde Buenos Aires solían pasar por otros puntos interiores como Córdoba y Santa Fe, también productoras de este animal (Moutoukias, 1988: 30-31). Algunos testimonios de la época pertenecientes al cabildo de Santa Fe sirven para ilustrar la importancia del mular a nivel comercial. En 1729, el organismo político en cuestión discutió sobre los arbitrios propuestos por el gobernador Bruno Mauricio de Zavala para cobrar sobre las cargas de las carretas y las mulas que entraban y salían de la jurisdicción, entre otras cosas como tabaco, azúcar y aguardientes (AGPSF, ACSF, Carpeta 14 "A" 73: 97-105b). Al año siguiente, decidieron mandar al teniente de gobernador a que revisara dia- 
riamente las entradas y salidas de carretas, mulas de carga y embarcaciones para tomar razón de las entradas y salidas (AGPSF, ACSF, Tomo X "A": 24-25b). Diez años más tarde lo encontramos exigiendo al arrendador de arbitrios de la ciudad que diera declaración jurada, entre otras cosas, de las mulas que habían entrado y salido de la jurisdicción (AGPSF, ACSF, Tomo XI: 7-8b). En definitiva, el gobierno local le daba importancia más que nada al control impositivo y de las transacciones comerciales que se hacían con los mulares.

Pese a la "atlantización" de la economía colonial que se viene mencionando en relación a la producción agropecuaria, cabe destacar que hasta las rebeliones indígenas del Alto Perú (a partir del decenio de 1780) y las guerras de Independencia, las relaciones con Potosí seguían teniendo un papel central en los flujos económicos y financieros del espacio, y la cría de mulas seguiría siendo de lo más importante para las UP (Garavaglia, 1999: 218; Paz, 1999: 47; Fradkin y Garavaglia, 2009: 87). Los inventarios, testamentos y tasaciones ponen en discusión estas posturas, o al menos dejan a la vista la importancia que fue adquiriendo la cría del vacuno desde lo numérico: eran 42 UP con ganado vacuno, mientras que las que practicaban la cría del híbrido eran 36, con un total de 13.109 animales y una densidad de 364, 13 por cada establecimiento. En cuanto al primer tipo de hacienda, este promedio es de 492,71 (un total de 20.694 cabezas distribuidas entre las 42 UP). En síntesis, si bien no se puede calcular la cantidad exacta de animales, sobre todo en el caso de las partidas de mulas, parece acertado sostener que ambos tipos de prácticas pecuarias eran muy relevantes, y que además se complementaban más que contraponerse: de los 58 que poseían ganado, 49 contaban al menos con vacas o relacionados al mular $(84,48 \%)$, mientras que en 31 coexistían ambas actividades (53,44\%) (AGN, Sucesiones). Parece que hacia mediados del siglo XVIII, las estancias de cría se estaban volcando a la diversificación de su producción hacia al menos 3 mercados: el "exterior" (exportación de cueros), el regional (envío de mulas en pie) y local (abasto de carne y derivados) (Garavaglia, 1996; Pelozatto Reilly, 2015a).

\section{El ovino: carne y producción textil}

Según los documentos consultados, el ganado ovino también estaba bastante difundido en los establecimientos de cría, y lo fue tanto desde el siglo XVII (González Lebrero, 1993), como hasta entrado el XIX (Mayo, 2004). De los 416 terrenos con ganado registrados en 1738, 67 criaban ovejas $(16,1 \%)$, siendo la cantidad total aproximada de 5.877 cabezas (87,71 animales por explotación). En 1744, una cantidad estimada de 6.611 ovejas se distribuían por las tierras de 19 empadronados ( $22 \%$ sobre todos los que tenían animales) que declararon tener acceso a dicho ganado (casi 
348 ovejas por UP) (ANH, Padrones de 1738 y 1744: 288-327; 509-709). Tomando los datos brindados por la fuente analizada restante, las tendencias son bastante similares: si bien este tipo de variante de la ganadería no estaba tan difundida en cuanto al número de unidades dedicadas a la misma (en 20 de los 58 establecimientos de cría, lo cual equivale a un $34,48 \%$ ), la concentración de cabezas por UP era enorme (566,4 ovinos por c/u aproximadamente) (AGN, Sucesiones).

La necesidad de criar estos animales estaba ligada al abasto de carne y la lana para la producción textil dentro de las unidades (Garavaglia, 1999: 218; Mayo, 2004, 2004: 41). Un rasgo particular para destacar esta clase de hacienda era su precio ínfimo por cabeza (Mayo, 2004: 41), y su aparición por lo general en planteles numerosos. Por ejemplo, en todos los casos registrados en Arrecifes (1738), las cantidades estaban entre 50 y 500 ovinos por establecimiento productivo (ANH, Padrón de 1738: 314-317). Hay casos para destacar, como el de Tomás de Arroyo, quien tenía estancia y chacra en el pago de Magdalena hacia 1744, y en donde se vieron supuestamente 3.000 ovejas (ANH, Padrón de 1744: 701). La mayoría de los criadores analizados a partir de las fuentes protocolares contaban con varias manadas muy grandes de esta especie: Petrona de Castro (1738) contaba, entre otras cosas, con 800. Entre los bienes de Esteban Correa (1742) fueron declaradas 1.197 cabezas (AGN, Sucesiones, 5336); Joseph de Esquivel (1744) tenía aproximadamente 400 en sus tierras del pago de las Conchas (región de chacras) (AGN, Sucesiones, 5671); el capitán Joseph López tenía 1.157 hacia el momento de su muerte en 1752 (AGN, Sucesiones, 6722). Miguel Cuello (1756) tenía 1.727 ovinos (AGN, Sucesiones, 5336). Unas 1.300 eran las que aparentemente estaban en propiedad de Tiburcio Casco (AGN, Sucesiones, 5338).

Esto se debía no únicamente a las salidas económicas que representaban las ovejas (carne y lana), sino fundamentalmente a su bajo precio per cápita: las ya mencionadas 800 ovejas de doña Castro fueron tasadas a 2 reales cada una. De entre los ovinos de Esteban Correa, había 36 que totalizaban 9 pesos entre todos (AGN, Sucesiones, 5336); entre las propiedades de Joseph Carrizo (1758), había 207 ovejas a 6 reales por cabeza (AGN, Sucesiones, 5337). Las 1.300 que poseía don Tiburcio Casco valía 2 reales cada una (AGN, Sucesiones, 5338). En pocas palabras, se trataba de ganados muy baratos si se comparan con los precios de las vacas (generalmente valían un peso de a 8 reales por cabeza, aunque las lecheras podían llegar a rondar los 18 reales), las yeguas (superaban siempre los 4 reales) y las mulas (siempre por encima de los 18 reales). Lamentablemente la documentación no permite divisar nítidamente lo que se hacía con los lanares en las chacras y estancias, más allá de algunos indicios como la presencia de prendas de lana. 


\section{Los animales característicos de la producción agrícola}

En importancia menor en cuanto a la cantidad dentro de las UP estaban los caballos y bueyes, dedicados principalmente a la producción agrícola y el transporte (Garavaglia, 1991; 1999). Los padrones rurales y sucesiones nos permiten reconstruir diferentes situaciones en cuanto a la tenencia de la tierra y las características territoriales de las UP. En 1738, se censaron 180 UP con caballos y 74 con bueyes, representando respectivamente el 43,26\% y el 17,78\% sobre el total de explotaciones con ganado (ANH, Padrón de 1738: 288-327). Lamentablemente la fuente no permite acercarse demasiado a las cantidades, que quedaron explícitas en apenas algunos casos.

En los inventarios y tasaciones se puede apreciar que estas bestias de tiro y carga eran empleados en distintos tipos de explotaciones. Don Miguel de Riblos tenía entre sus distintas tierras de estancia en Areco varios bueyes y caballos, junto con enormes planteles de vacunos, yeguas y mulas, por lejos los más abundantes (AGN, Sucesiones, 8122). Joseph de Esquivel complementaba la cría de bueyes (tenía 5), con las de terneras y vacas a pequeña escala (sumaban 4), al mismo tiempo que practicaba la agricultura del trigo (AGN, Sucesiones, 5671). El capitán Marcos Rodríguez (1740), tenía 25 caballos entre sus haciendas, lo cual hace pensar que no solamente eran empleados en el sector agrario y el comercio en carretas, sino también como parte del proceso de cría de mulas (tenía muchas yeguas de cría y algunos burros hechores) (AGN, Sucesiones, 8130). Por su parte, Juan Manuel Arce tenía bueyes y caballos en sus tierras de chacra junto con carretas y carretones, aunque también habia espacio para la cría de mulas. Hay otros casos en donde las cantidades son más abundantes, pero con vínculos claros con el cereal y el comercio, como por ejemplo el establecimiento de doña María Ayala (1751), donde había 12 bueyes y 28 potrancas junto a varias carretas (AGN, Sucesiones, 3859). Otros casos, en cambio, parecen mostrar la figura de lo que se asemeja a lo que podríamos llamar criadores animales de carga y tracción a gran escala: don Lorenzo Rodríguez, para citar un ejemplo bastante particular, tenía 102 caballos y 51 bueyes. Sin embargo, en el mismo establecimiento de cría se registraron 1.508 cabezas entre vacas, novillos y terneras, más otras especies: 9 mulas mansas, 4 yeguas madrinas, 8 manadas de yeguas de cría y 216 ovejas (AGN, Sucesiones, 8130).

En definitiva, el interés por los animales analizados en este apartado puede estar vinculado a la venta de los mismos, a las tareas agrícolas (se hallaron en la misma estancia hoces, azadas, un molino de tiro y carretones) y en el caso de los equinos, a la fabricación de híbridos. Ejemplos como el de Lorenzo Rodríguez permiten pasar cuestiones a tratar en el siguiente punto de esta investigación: ¿era la ganadería especializada o diversificada?, ¿qué relación 
existía con las prácticas agrícolas? Preguntas que se intentarán responder a continuación.

\section{Ganadería diversificada y establecimientos mixtos}

Uno de los objetivos principales de este artículo está relacionado en caracterizar la producción interna de las UP de cría de ganado y las diferentes alternativas mercantiles posibles para los productores pecuarios. Asimismo, se busca ingresar en una vieja discusión de la historiografía colonial rioplatense: si existía o no complementariedad entre la ganadería y la agricultura.

Las fuentes consultadas son contundentes en cuanto al carácter diversificado de la ganadería: en 1738, se contaron 1.023 unidades en total, de las cuales 416 poseían ganado de algún tipo (40,66\%), entre las cuales 244 tenían ganado vacuno $(58,65 \%)$ y 183 contaban con ese tipo de recurso pecuario más otras especies (representaban el $44 \%$ entre todas las extensiones donde había cría). En 1744, el total de explotaciones fue de 891, de las cuales 104 poseían animales (apenas un 11,67\%), entre las que había 86 que se dedicaban de alguna forma u otra al vacuno (82,69\%), mientras que 37 pueden ser definidas como "mixtas" (43\% de los sitios con hacienda) (ANH, Padrones de 1738 y 1744: 288-327; 509-709). Considerando las propiedades de los vecinos que fueron analizadas (81 en total), vale destacar que 58 tenían ganado $(71,6 \%$ incluyendo solamente las unidades en donde aparecen explícitamente los animales), y entre éstos, 42 criaban vacunos $(72,41 \%)$ en sus diferentes formas, como vacas, toros, novillos, terneras y vacas lecheras. Por otra parte, 36 era el total de las que tenían ganados vinculados a la producción de mulas, incluyendo estas últimas (62\% sobre el total de establecimientos con cría). Otras 31 UP complementaban la cría de vacunos y mulares $(53,44 \%)$.

Incluso existen varios ejemplos puntuales de explotaciones que incluían todos los tipos de ganado analizados en esta investigación y en cantidades variables. El caso más antiguo encontrado es el del capitán don Dionisio Chiclana (1726), que poseía 16 mulas mansas (valuadas en 99 pesos total), 66 yeguas madrinas a un peso cada una, 81 vacas de rodeo que sumaban 81 pesos en dinero, 110 bueyes (440 pesos), 162 caballos de 2 pesos por cabeza y 320 de 4 pesos (AGN, Sucesiones, 5335). Entre los bienes de las estancias que habían sido de don Miguel de Riblos, en el pago de Areco, se hallaron 14 bueyes, 45 caballos, 53 burros, 1.216 vacunos (entre vacas y terneros), 1.360 mulas y 4.935 yeguas (AGN, Sucesiones, 8122). Don Felipe Castro (1729) tenía bajo sus dominios 11 burros, 20 bueyes, 220 entre potros y caballos, 811 yeguas de vientre y 1.511 cabezas entre vacas y terneras (AGN, Sucesiones, 5335). Manuel de la Cruz, vecino de Luján censado en 1738, declaró 1.000 cabezas de vacuno, además de tener planteles de bueyes, caballos y yeguas. 
Antonio Gelves, de Arrecifes, totalizó 500 vacas, 300 yeguas, 30 caballos y 400 ovejas. Don Bernardo Quintana, vecino del mismo pago, acusaba tener 1.000 yeguas, 300 vacunos, 200 ovinos y 20 equinos (ANH, Padrón de 1738: 313-318). En 1744, Pedro Barragán, vecino de Magdalena, fue encontrado con 1.500 ovejas, 1.000 vacas y 200 yeguas de cría (ANH, Padrón de 1744: 705). El capitán Francisco Cordero, fallecido en 1746, acumulaba entre sus bienes 663 entre vacas y terneras, 212 bueyes, 76 yeguas, 33 caballos y 31 mulas (AGN, Sucesiones, 5336). Podrían seguir citándose casos, pero no es la intención de esta investigación, sino demostrar que la coexistencia entre distintos tipos de ganado en las UP de Buenos Aires era una tendencia muy clara en el período analizado, según lo demuestran las cifras y los ejemplos descriptos.

Un rasgo que se puede apreciar es la complementariedad entre ganadería y agricultura, vinculada esta última fundamentalmente a la producción de cereales. Un indicador de esta relación puede ser, en primer lugar, la presencia de tierras de chacras en donde había varias especies de ganado, además de las señaladas como vitales para el funcionamiento de las tareas agrícolas (bueyes y caballos): en 1738, el capitán Martín Peredo tenía ovejas en su chacra de "Conchas arriba", mientras que el capitán Bartolomé Verdún contaba con bueyes y caballos en un establecimiento de la misma clasificación (ANH, Padrón de 1738: 309). El teniente don Julio Fredes, de Los Arroyos, tenía en sus tierras de chacra 6 caballos y 4 bueyes, al igual que Joseph Acasuso, del mismo pago. Por otra parte, se encontraron en aquel paraje otras 31 chacras, de las cuales 6 tenían ganado, tratándose siempre de caballos y bueyes (ANH, Padrón de 1738: 320-322). En 1744 también se registraron casos, como el de Pedro López, quien tenía estancia y chacra en La Matanza, donde criaba vacas, al igual que su vecino Diego de Videla, quien se dedicaba a las sementeras y la cría de lecheras. Por su parte, Martín Gutiérrez, chacarero de la zona, contaba con un rodeo de vacas en sus tierras. Tomás de Arroyo, de Magdalena, tenía 3.000 ovinos y 1.500 vacunos entre sus estancias y chacras. En las tierras de chacra pertenecientes a Francisco Basurco en el mismo pago, se encontraron 150 cabezas de ganado vacuno (ANH, Padrón de 1744: 671-708).

Otro indicio de complementariedad es la existencia de herramientas agrícolas y trigo en los establecimientos de cría. De los 58 establecimientos identificados con la cría de animales, 44 (75,86\%) poseían azadas, hoces, molinos, atahonas, arados, fanegas de trigo y/o maíz. En síntesis, la gran mayoría de las UP complementaba la ganadería y sus distintas variantes con la producción de cereales. Esto puede verse tanto en casos de pequeños y medianos productores, como Francisco Lerma (1759), quien tenía 4 yuntas de bueyes, 3 caballos, 2 yeguas madrinas, 5 cueros de toro, 16 gallinas, un gallo y pollos, además de 9 fanegas de trigo almacenadas, una huerta, 
azadas grandes y un mortero viejo (AGN, Sucesiones, 6723). Y también entre los hacendados-terratenientes más poderosos de la época, como lo fuera el capitán Ramón López (1748), estanciero de La Matanza que ostentaba 3.675 animales (entre vacas, terneras y lecheras), 170 yeguas, 8 mulas y 6 bueyes mansos sobre 500 varas de frente de tierras. Además le hallaron 20 cueros, 5 fanegas de trigo almacenado, piezas de grasa y sebo, un carretón, 2 carretas, más otras manadas de yeguas y vacas (AGN, Sucesiones, 6722). Casos como los citados en este apartado responderían a varios mercados como el abasto de carne, sebo y grasa, la exportación de cueros, el comercio de mulas, y la producción de trigo para el consumo y mercado local (Halperín Donghi, 1993: 68; Garavaglia, 1999: 216-218; Mayo, 2004: 40-41).

\section{Conclusiones}

Luego de la lectura y el análisis descriptivo y cuantitativo que se hizo sobre los padrones rurales y el corpus de distintos documentos correspondientes a las sucesiones, se han alcanzado algunas conclusiones tanto sobre la evolución de las características del ganado vacuno, como acerca del carácter diversificado de la producción pecuaria y la existencia de una complementariedad más que un contraste entre la ganadería y la agricultura: a) la mayoría de los establecimientos registrados por fuentes protocolares tenían algún tipo de ganado como mínimo; b) la ganadería vacuna fue aumentando en cuanto al número de establecimientos y la concentración de animales de este tipo dentro de los mismos; c) la misma tenía varias alternativas comerciales como la exportación de cueros (que creció y se intensificó durante el período), el abasto de carne local (una preocupación central para vecinos y autoridades locales), y la elaboración de otros productos como sebo y grasa; d) existía una relación de complementación entre la cría de vacas y la producción de mulas, sobre todo en las grandes UP, que de esa manera se vinculaban -además de los mercados ya señalados- al Alto Perú minero; e) había cría de otras especies como las ovejas, en un número de explotaciones menor al de los anteriores, y la concentración por establecimiento era mucho más alta; f) había otros tipos de hacienda que estaban estrechamente vinculados a la producción agrícola, como los bueyes (mantenidos específicamente para el arado, la carga, el transporte y la molienda) y los caballos (que también formaban parte del proceso de producción de mulares, sobre todo cuando los encontramos en cantidades más abundantes de lo normal), g) la ganadería y sus diversas formas se encontraban emparentadas con la agricultura del cereal, que representaban no solamente alimento para los habitantes y trabajadores de las UP (los panificados eran uno de los dos alimentos más presentes en la dieta de los porteños, junto con la carne de vaca), sino también como forraje para los animales y productos comercializables en el mercado de la ciudad. 
Resumiendo, lejos estaba la ganadería vacuna de ser algo despreciable en las UP de cría, y la misma fue ganando peso en las mismas y en los mercados coloniales durante la primera mitad del siglo XVIII, como parte de la "atlantización" de la economía colonial y del estallido que se produjo en la exportación de pieles de toro (Milletich, 2000; Jumar, 2008). Asimismo, el visible aumento de la población, sobre todo desde la década de 1740, condujo a la necesidad de más carne para el abastecimiento local, que bien pudo haber sido otro disparador para la cría de vacunos (Garavaglia, 1996; Mayo, 2004; Pelozatto Reilly, 2015a). Sin embargo, también existieron otras alternativas para la ganadería, no solamente las vinculadas a este tipo de rama, sino también con la cría de mulas (que siguió siendo una de las dos actividades más importantes, por su relevancia para el abastecimiento de los centros productores de plata del Virreinato del Perú), ovejas (carne para consumo y mercado local, más lana para distintas fabricaciones de textiles artesanales) u otros como los bovinos y equinos que eran empleados como fuerza de trabajo, de carga y en los vehículos de transporte. La agricultura del cereal estaba muy difundida en las UP, mismo en las que tenían hasta grandes planteles de ganado y se orientaban hacia sus respectivos mercados, y no solamente para el consumo interno de los trabajadores y animales, sino también con salida a la demanda de la ciudad y su campaña.

Es preciso seguir desarrollando en otras investigaciones profundas otras problemáticas vinculadas a las unidades de producción rurales, como las condiciones de vida y trabajo, los distintos tipos de mano de obra que se utilizaban, cómo se fueron configurando diversos vínculos sociales en torno a las tierras y el ganado, entre otras que quedan abiertas a discusión.

\section{Referencias Bibliográficas}

\section{Fuentes primarias}

\section{a) Documentales}

Academia Nacional de la Historia (ANH). Padrón de 1738 y Padrón de 1744.

Archivo General de la Nación (AGN). Sucesiones.

Archivo General de la Nación. Acuerdos del Extinguido Cabildo de Buenos Aires (AECBA).

Archivo General de la Provincia de Santa Fe (AGPSF). Actas del Cabildo de Santa Fe (ACSF). 


\section{Fuentes secundarias}

\section{a) Artículos}

Birocco, C. M. (1996). "Historia de un latifundio rioplatense: las estancias de Riblos en Areco, 1713-1813", en Anuario de Estudios Americanos, Vol. 53, № 1, pp. 73-99.

Birocco, C. M. (2003). "Alcaldes, capitanes de navío y huérfanas. El comercio de cueros y la beneficencia pública en Buenos Aires a comienzos del siglo XVIII", ponencia presentada en las III Jornadas Internacionales de Historia Económica, Asociación Uruguaya de Historia Económica (AUDHE).

Dupuy, A. (2010). "El estanco de carne y su crisis en Buenos Aires colonial desde una visión comparativa", en Revista Brasileira de História, Vol. 30, № 60, pp. 211-231.

Fradkin, R. (1993). "Producción y arrendamiento de Buenos Aires del siglo XVIII: la hacienda de la Chacarita (1779-84)", en Fradkin, R. (Compilador). La historia agraria del Río de la Plata colonial. Los establecimientos productivos (II). Buenos Aires: Centro Editor de América Latina, pp. 40-69.

Fradkin, R. (2000). "El mundo rural colonial", en Tandeter, E. (Director). Nueva Historia Argentina. Tomo Il: la sociedad colonial". Buenos Aires: Editorial Sudamericana, pp. 241-284.

Garavaglia, J. C. (1991). "El pan de cada día: el mercado del trigo en Buenos Aires, 1700-1820", en Boletín del Instituto de Historia Argentina y Americana "Dr. E. Ravignani", Tercera Serie, No 4, pp. 7-29.

Garavaglia, J. C. (1993). "Las estancias en la campaña de Buenos Aires. Los medios de producción (1750-1850)", en Fradkin, R. (Compilador). La historia agraria del Río de la Plata colonial. Los establecimientos productivos (II). Buenos Aires: Centro Editor de América Latina, pp. 124-208.

Garavaglia, J. C. (1994). "De la carne al cuero. Los mercados para los productos pecuarios (Buenos Aires y su campaña, 1700-1825", en Anuario del IEHS, pp. 61-96.

Gelman, J. D. (1993). "Nuevas perspectivas sobre un viejo problema y una misma fuente: el gaucho y la historia rural del Río de la Plata colonial", en Fradkin, R. (Compilador). La historia agraria del Río de la Plata colonial. Los establecimientos productivos (I). Buenos Aires: Centro Editor de América Latina, pp. 121-142.

Gelman, J. D. (1993b). "Una región y una chacra en la campaña rioplatense: las condiciones de la producción triguera a fines de la época colonial", La historia agraria del Río de la Plata colonial. Los establecimientos productivos (II). Buenos Aires: Centro Editor de América Latina, pp. 7-39. 
González Lebrero, R. E. (1993). "Chacras y estancias en Buenos Aires a principios del siglo XVII", en Fradkin, R. (Compilador). La historia agraria del Río de la Plata colonial. Los establecimientos productivos (II). Buenos Aires: Centro Editor de América Latina, pp. 70-123.

Halperín Donghi, T. (1993). "Una estancia en la campaña de Buenos Aires, Fontezuela, 1753-1809", La historia agraria del Río de la Plata colonial. Los establecimientos productivos (I). Buenos Aires: Centro Editor de América Latina, pp. 45-65.

Harari, E. F. (2003). "Las vaquerías a comienzos del siglo XVIII: una aproximación desde el marxismo", ponencia presentada en las III Jornadas Internacionales de Historia Económica, Asociación Uruguaya de Historia Económica (AUDHE).

Jumar, F. (2008). "El primer boom de la exportación de cueros y la sociedad local. Río de la Plata. Fines del siglo XVII, comienzos del siglo XVIII", ponencia presentada en XXI Jornadas de Historia Económica, Asociación Argentina de Historia Económica, Universidad Nacional de Tres de Febrero.

Mayo, C. y Fernández, A. (1993). "Anatomía de la estancia colonial bonaerense (1750-1810) ", La historia agraria del Río de la Plata colonial. Los establecimientos productivos (I). Buenos Aires: Centro Editor de América Latina, pp. 67-82.

Milletich, V. (2000). "El Río de la Plata en la economía colonial", en Tandeter, E. (Director). Nueva Historia Argentina. Tomo Il: la sociedad colonial". Buenos Aires: Editorial Sudamericana, pp. 189-240.

Pelozatto Reilly, M. L. (2015). "El Cabildo de Buenos Aires y la economía rural: las recogidas de ganado y el abasto dentro de su jurisdicción, 1723-1750", en Carta Informativa XXXVII de la Junta de Estudios Históricos de La Matanza, Universidad Nacional de La Matanza, pp. 7-42.

Pelozatto Reilly, M. L. (2015b). "El Cabildo, los vecinos y la utilización de la 'otra banda' como territorio alternativo en la economía rural colonial. Buenos Aires y Santa Fe durante la extinción del ganado cimarrón y las vaquerías tradicionales (1720-1750)", en Estudios Históricos, año VII, No 14, Centro de Documentación Histórica del Río de la Plata y Brasil "Dr. Walter Rela", Uruguay.

Salvatore, R. y Brown, J. (1993). "Comercio y proletarización en la Banda Oriental tardo-colonial: la estancia de Las Vacas, 1791-1805", en Fradkin, R. (Compilador). La historia agraria del Río de la Plata colonial. Los establecimientos productivos (I). Buenos Aires: Centro Editor de América Latina, pp. 83-120.

\section{b) Libros}

Azcuy Ameghino, E. (1995). El latifundio y la gran propiedad colonial rioplatense. Buenos Aires: Fernando García Cambeiro. 
Birocco, C. M. (2015). La élite de poder en Buenos Aires colonial: cabildo y cabildantes entre los Habsburgo y los Borbones (1690-1726), Tesis de posgrado presentada para la obtención del grado de Doctor en Historia. Universidad Nacional de La Plata, Facultad de Humanidades y Ciencias de la Educación.

Fradkin, R. y Garavaglia, J. C. (2009). La Argentina colonial. El Río de la Plata entre los siglos XVI y XIX. Buenos Aires: Siglo XXI Editores.

Garavaglia, J. C. (1999). Pastores y labradores de Buenos Aires. Una historia agraria de la campaña bonaerense 1700-1830. Buenos Aires: Ediciones de la Flor.

Halperín Donghi, T. (2010). Historia contemporánea de América Latina. Buenos Aires: Alianza Editorial.

Mayo, C. (2004). Estancia y sociedad en la pampa (1740-1820). Buenos Aires: Editorial Biblos. 
